\title{
राज्यसमाजवाद आणि कल्याणकारी राज्य
}

State socialism and welfare state

\section{Dr. Rakshit Madan Bagde,}

Assistant Professor,Late. Mansaramji Padole Arts College, Ganeshpur Bhandara

ORCID iD - 0000-0002-7507-0244

Web of Science ResearcherID - AAF-2760-2020

SSRN - Author ID: 4770534

Authenticus ID - R-00J-YM2

Vidwan-ID : 221858

rakshitbagde@gmail.com

डॉ. आंबेडकरांना वाटत असे की, भारताच्या आर्थिक उन्नतीसाठी दारिद्रच व विषमता निर्मुलन आणि बहुजन समाजाची शोषणमुक्तता यावर भर दिला पाहिजे. 1930 व 1940 या काळात व पुढेही काही वर्षे त्यांनी आपल्या लेखनात व भाषणात बहुजन समाजाच्या शोषणमुक्ततेवर भर दिला. डॉ. आंबेडकरांना समाजवाद हवा होता, परंतु पारंपारिक समाजवाद त्यांना पसंत नव्हता. त्यांच्या दृष्टिकोणातून श्रमीक आणि शोषित वर्गाच्या आर्थिक सुरक्षेकडे अधिक लक्ष देऊन योजना तयार करायला पाहिजे. नैतिक प्रेरणा लोकांमध्ये असायला पाहिजे, आणि त्यांनी मिळून जे काही आपल्या श्रमाने कमविले ती सर्व संपत्ती समान रूपात वाटली पाहिजे.

डॉ. आंबेडकरांनी राज्ये आणि अल्पसंख्यांक या शीर्षकाखाली एक पुस्तीका 1947 साली प्रसिद्ध केली. यात मूळ अधिकार, अल्पसंख्यांकांचे अधिकार आणि अनुसूचित जातींच्या सुरक्षा उपायांची माहिती दिली आहे.

राज्यसमाजवादातील तरतुदी :-

डॉ. आंबेडकरांच्या राज्यसमाजवादात पुढील तरतुदींचा समावेश होता.

* शेती हा राज्याचा उद्योग असावा.

* विमा, उद्योग आणि जमीन ज्यांनी धारण केल्या आहेत त्या ताब्यात घेण्याचा अधिकार सरकारला असावा. त्यांना कर्जरोख्यांच्या स्वरूपात मोबदला द्यावा. 
कर्जरोखे धारकांना रोख रक्कम केव्हा व कशा प्रकारे घ्यावी, हे राज्य ठरवील.

* कर्जरोख्यावर वरसा हक्क लावता येईल.

* कर्जरोखे धारकांना शासकीय नियमाने रोख रक्कम मिळेल.

* शेती व्यवसाय खालील प्रमाणे संगठीत केला जाईल -

अ) शासन शेतीचे योग्य भाग करून ते कसायला उपलब्ध करेल.

i) जमिनीवर सामुहिक शेती केली जाईल.

ii) शासकीय नियमाने शेती केली जाईल.

iii) शेती उत्पादकांनी ठरविल्या पद्धतीप्रमाणे कसणान्या प्रत्येकास

शेतीच्या मालात सवलतीचे पैसे देऊन सहभागी होता येईल.

ब) जमीन खेड्यातील लोकांना भाङ्याने देण्यात येईल. ती देताना जात, पंथ असा भेद न करता दिली जाईल.

क) सामुहिक शेतीसाठी पैसे, पाणी, जनावरे, खते आणि बी इ. उपलब्ध करून देणे शासनावर बंधनकारक राहील.

ड) i) राज्य शेतीसाठी सवलती देवू शकतो.

अ) शेतसान्याचा काही भाग.

ब) कर्जधारकाला द्यावयाचा काही भाग.

क) साधनांसाठी दिलेल्या पैशाचा काही भाग.

ii) सामुहिक शेतीच्या योजनेला बाधक होईल असे आचरण केले तर त्याला कोणता दंड द्यावा हे शासनाने ठरवावे. 
* जे प्रमुख उद्योग आहेत ते राज्याच्या मालकीचे असावेत व ते राज्याने चालवावेत.

* जे प्रमुख उद्योग नाहीत पण पायाभूत आहेत ते राज्याने चालवावे किंवा महामंडळाद्वारे चालवावे.

* विमा क्षेत्रात राज्याची मक्तेदारी असावी आणि राज्याने प्रत्येक प्रोढाला त्याच्या उत्पन्नाच्या प्रमाणात तो घेण्यास भाग पाडावे.

* ही योजना जितक्या लवकर होईल तितक्या लवकर अंमलात आणावी परंतु घटना अंमलात आल्यापासून दहा-वर्षापेक्षा अधिक काळ होता कामा नये

डॉ. आंबेडकरांनी घटना समितीला सादर केलेले निवेदन हे राज्यसमाजवादाचे प्रारूप होते. राज्य घटनेतच समाजवादाची तरतुद असावी असे त्यांचे मत होते. संविधानाच्या उद्देशिकेत आणि निर्देशक तत्त्वात डॉ. आंबेडकरांनी समाजवादाचा समावेश केलेला आहे. ज्यात बुद्ध तत्त्वज्ञानाच्या छटा स्पष्टपणे दिसून येतात. डॉ. आंबेडकरांचे आर्थिक धोरण म्हणजे आर्थिकदृष्ट्या कल्याणकारी राज्याच्या निर्मितीला साह्यभूत ठरणारे 'समृद्धीचे अर्थकारण' आणि सामाजिकदृष्ट्या समतावादी समाजरचनेच्या प्रस्थापनेला गती देणारे 'समाजोद्धाराचे अर्थकारण व कल्याणकारी राज्याचे अर्थशासत्र' आहे.

संदर्भ -

जाधव नरेद्र (1992) - डॉ. आंबेडकर आर्थिक विचार आणि तत्त्वज्ञान, सुगावा प्रकाशन पुणे,

आजाद रामगोपाल, (2003) . अनुवाद : 'राज्य और अल्पसंख्यांक दलित', समता प्रकाशन -समाता सैनिक दल, लष्करीबाग, नागपूर, 23 फेब्रुवारी.

खिमेसरा ज्ञानचन्द्र (1995) - डॉ. आंबेडकर का आर्थिक चिंतन, मध्यप्रदेश हिन्दी ग्रथ अकादमी, भोपाल, 